\title{
Preoperative Prognostic Nutritional Index Value is Related to Postoperative Delirium in Elderly Patients After Noncardiac Surgery: A Retrospective Cohort Study
}

This article was published in the following Dove Press journal:

Risk Management and Healthcare Policy

He Liu, (1D) ${ }^{1,2, *}$

Mingsheng Dai, (D) ${ }^{1,3, *}$ Huilian Guan,' Xing Gao,' Yang Zhou,' Xun Sun, (D) Jian Zhou,' Xiaoyi Hu,' Xiang Li,' Yu Song,' Yuan Han, ${ }^{4}$ Junli Cao $\mathbb{D}^{1,2}$

'Department of Anesthesiology, The Affiliated Hospital of Xuzhou Medical University, Xuzhou City 221002, Jiangsu Province, People's Republic of China; ${ }^{2}$ Jiangsu Province Key Laboratory of Anesthesiology, Xuzhou Medical University, Xuzhou City 221004, Jiangsu Province, People's Republic of China; ${ }^{3}$ Department of Anesthesiology, Union Hospital, Tongji Medical College, Huazhong University of Science and Technology, Wuhan, Hubei, People's Republic of China; ${ }^{4}$ Department of Anesthesiology, Eye \& ENT Hospital of Fudan University, Shanghai 2003I, People's Republic of China

*These authors contributed equally to this work

Correspondence: Junli Cao Jiangsu Province Key Laboratory of Anesthesiology, Xuzhou Medical University, NO. 209 Tongshan Road, Yunlong District, Xuzhou City 221004, Jiangsu Province, People's Republic of China

$\mathrm{Tel} / \mathrm{Fax}+86-051$ 6-83262686

Email caoj10310@aliyun.com

Yuan Han

Department of Anesthesiology, Eye \& ENT Hospital of Fudan University, 83 Fenyang Road, Shanghai 20003I, People's Republic of China

Email hanyuan2002@I63.com
Purpose: Malnutrition has been considered as a risk factor for postoperative delirium (POD). The Prognostic Nutritional Index (PNI) is a validated tool for assessing nutritional status. This study aimed to investigate the association between preoperative PNI values and the occurrence of POD in elderly surgical patients.

Methods: The retrospective cohort study included 361 elderly individuals who underwent noncardiac surgery between 2018 and 2019. Perioperative data were collected from the patients' medical records. PNI was used to evaluate preoperative nutritional status. The primary outcome was the occurrence of POD. Univariate and multivariate logistic regression analyses were used to identify key factors associated with POD and assess the relationship between PNI values and the occurrence of POD. Receiver operating characteristic (ROC) curve analysis was used to assess the predictive value of PNI for POD.

Results: Seventy-two (19.9\%) individuals developed postoperative delirium after surgery. Compared with patients of normal nutrition status (PNI $\geq 50$ ), mild malnutrition (PNI 45-50) did not increase the risk of POD, while patients with moderate to severe malnutrition (PNI 40-45) (odds ratio [OR], 2.92; 95\% confidence interval [CI], 1.31-6.50) and serious malnutrition (PNI < 40) (OR, 3.15; 95\% CI, 1.12-8.83) were more likely to develop POD. The cut-off value of PNI was 46.05 by ROC curve analysis, the area under the curve (AUC) was 0.69 (95\% CI 0.62-0.77).

Conclusion: Preoperative PNI value is related to postoperative delirium in elderly patients after noncardiac surgery.

Keywords: noncardiac surgery, elderly patients, nutritional status, prognostic nutritional index, postoperative delirium

\section{Introduction}

Postoperative delirium (POD) is one of the most common neurological complications among elderly patients after surgery. Acute and fluctuating disturbance of consciousness, inattention, disorganized thinking and altered consciousness are characteristics of POD. ${ }^{1}$ POD is associated with several negative outcomes, such as longer length of stay in hospital, impaired functional abilities, increased longterm care requirement and mortality. ${ }^{2,3}$ It is generally believed that not only posoperative status, but also pre-existing factors, such as reduced functional status, advanced age, malnutrition and decreased cognitive levels, may be closely related to POD. ${ }^{4}$ 
Among the elderly, malnutrition commonly occurs, especially in those who are chronically ill or hospitalized. ${ }^{5}$ It was reported that the incidence of malnutrition ranges from $50 \%$ to $80 \%$ related to different types of disease. ${ }^{6,7}$ Poor nutritional status of surgical patients is associated with various adverse outcomes, including functional and cognitive impairment, and increased risk of depression. ${ }^{8-10}$ In clinical settings, several measures can reflect the status of nutrition, for instance, the Malnutrition Universal Screening Tool (MUST), ${ }^{11}$ Controlling Nutritional Status (CONUT), ${ }^{12}$ Short Form Mini Nutritional Assessment (MNA-SF), ${ }^{13}$ Geriatric Nutritional Risk Index (GNRI) ${ }^{14}$ and Prognostic Nutritional Index (PNI). ${ }^{15}$ Among these tools, the PNI is a convenient and accurate way to quantify nutritional status. Although two studies have shown that preoperative low PNI is related to increased risk of POD among patients undergoing orthopedic surgeries, ${ }^{16,17}$ the relationship between PNI and POD in elderly patients undergoing noncardiac surgeries is unclear.

Thus, we conducted the present retrospective study. The study may provide further evidence to support the idea of reducing the incidence of POD by strengthening patients' preoperative nutritional status, especially for elderly patients undergoing elective surgery.

\section{Materials and Methods}

\section{Study Design}

We conducted the retrospective study at the Affiliated Hospital of Xuzhou Medical University (Xu Zhou, China) between December 2018 and August 2019. The study was approved by the clinical research ethics committee of the Affiliated Hospital of Xuzhou Medical University (Certification No. XYFY2019-KL198-01, approval date: October 28, 2019), and registered at Chinese Clinical Trial Registry (ChiCTR2000029657, February 9, 2020). The ethics committee agreed that informed patient consent was not required for the retrospective and observational nature of this analysis. There was compliance with the 1964 Helsinki declaration. The information provided from patients' recorded data was kept confidential. Codes instead of names were used to identify the study populations. Inclusion criteria were as follows: (1) age 60 years or older; (2) American Society of Anesthesiologists (ASA) physical status I-III; (3) patients who underwent noncardiac surgery under general anesthesia. Exclusion criteria were as follows: Mini Mental State Examination (MMSE) scores less than 15, the duration of surgery less than 90 minutes, length of stay after surgery less than 3 days, preoperative albumin infusion, pre-existing neurological diseases (Parkinson's disease, Alzheimer's disease, or preoperative delirium), missing data or disconnection of follow-up.

\section{Data Collection}

The baseline characteristics and demographic details were obtained from the hospital medical information system. Preoperative, perioperative and postoperative data were collected, including age, gender, years of education, body mass index (BMI), smoking and drinking history, ASA physical status, MMSE score, ${ }^{18}$ pre-existing comorbidities, Charlson Comorbidity Index (CCI) ${ }^{19}$ and laboratory results, such as serum potassium levels, blood glucose, alanine transaminase (ALT), aspartate transaminase (AST), blood urea nitrogen (BUN), creatinine (Cr), hemoglobin, albumin, and lymphocyte count.

\section{Prognostic Nutritional Index Determination}

Nutritional status was assessed by the PNI. PNI was calculated as $10 \times$ albumin $(\mathrm{g} / \mathrm{dl})+0.005 \times$ total lymphocyte count (per $\mathrm{mm}^{3}$ ), and nutritional status was divided into 4 grades based on PNI value: normal (PNI $\geq 50$ ), mild malnutrition (PNI 45-50), moderate to severe malnutrition (PNI 40-45), serious malnutrition $(\mathrm{PNI}<40){ }^{20}$

\section{Delirium Assessment}

Delirium was assessed using rigorous methodologies, including the Confusion Assessment Method (CAM) ${ }^{21}$ applied to assess POD in wards, and the CAM for the intensive care unit (CAM-ICU) ${ }^{22}$ used in the PACU or the ICU. Patients were assessed for delirium at least $2 \mathrm{~h}$ after the end of surgery and twice-daily on the first 3 postoperative days at a minimum 6-hour interval. ${ }^{23}$ Additionally, investigators collected evidence of delirium from nurses, caregivers and medical records, including confusion, agitation, sedation, hallucinations and delusions.

\section{Primary Outcomes}

The primary outcome was the presence of POD during the first 3 days after surgery. The primary objective of this study was to evaluate the association between preoperative PNI value and the present of POD.

\section{Statistical Analysis}

The data were analyzed using SPSS software (version 19.0; SPSS Inc.; Chicago, IL, USA) and Prism (version 8.0.1; GraphPad Software, Inc.; USA). Continuous data were 
expressed as mean and standard error (SD) or as median and interquartile range (IQR), and analyzed by Mann-Whitney $U$-test or $t$-test, while categorical data were demonstrated as number (n, \%) and analyzed by Chi-square test or Fisher's exact test. Univariable and multivariable logistic regression models were estimated to identify independent risk factors of POD. Variables with $P<0.1$ ( 2 sided) in the univariable analysis were included in the multivariable regression model using a backward selection algorithm. Multicollinearity diagnostic was performed between the variables to evaluate the validity of the regression model by calculating the values of tolerance and the variance inflation factor (1/tolerance). Receiver operating characteristic (ROC) curve analysis was used to evaluate the predictive and cut-off value of PNI for POD. All $P$ values given are based on 2-tailed tests, and a $P$-value $<0.05$ was considered a statistically significant difference.

\section{Results}

\section{Enrollment}

A total of 650 consecutive patients were screened between December 2018 and August 2019; 250 patients did not meet the inclusion criteria, and 39 patients were with incomplete data. These patients were excluded from the study. Thus, 361 patients were enrolled in the study, and the records of the patients were available for the final analysis. The specific content is shown in the flow chart (Figure 1).

\section{Patient Characteristics}

A total of 72 cases (19.9\%) developed delirium in the first 3 days after surgery. Table 1 shows the demographic and perioperative data of these patients with or without POD, and no statistical difference was found in the smoking and drinking habits, the history of diabetes and hypertension, CCI, types of operation, operative blood loss, the volume of perioperative blood and fluid transfusion, the duration of operation and anesthesia between the patients with and without POD $(P>0.005)$ (Table 1).

The age of the patients in the POD group was significantly higher $(P=0.001)$, while the BMI $(P=0.003)$ and the MMSE scores $(P=0.002)$ were significantly lower than in the non-POD group. Furthermore, compared with patients of ASA II, the incidence of POD was higher in patients of ASA III $(P=0.022)$.

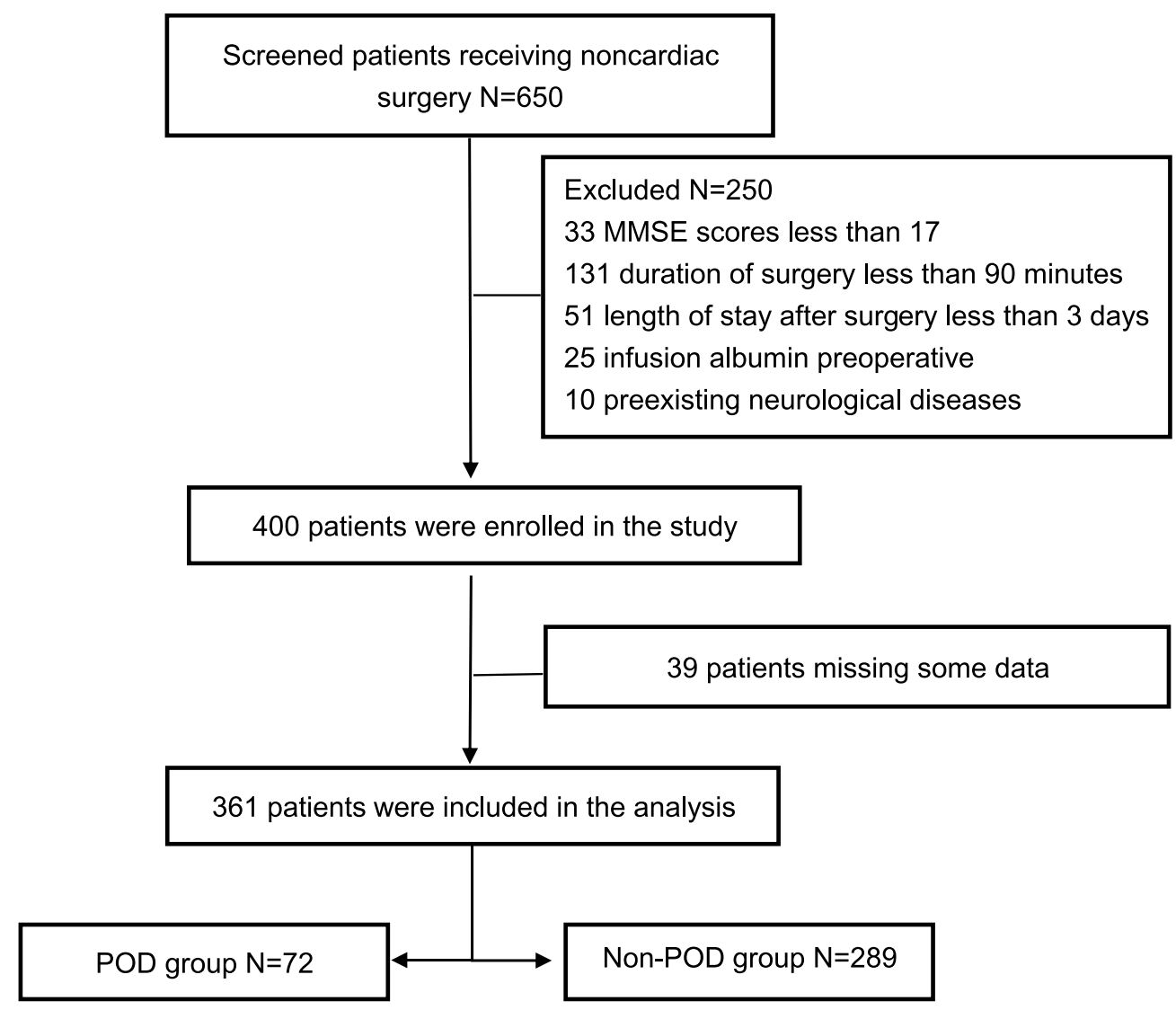

Figure I Flow chart of the patients. 
Table I Baseline and Perioperative Characteristics of Patients with POD and Non-POD

\begin{tabular}{|c|c|c|c|}
\hline & POD (n=72) & Non-POD (n=289) & $\boldsymbol{P}$ \\
\hline Male sex, n (\%) & $43(59.7)$ & $168(58.1)$ & 0.806 \\
\hline Age, year median (IQR) & $70(67,77)$ & $68(64,73)$ & $0.001 *$ \\
\hline Education, year median (IQR) & $5(0,7)$ & $5(0,7)$ & 0.944 \\
\hline ASA physical status, $n$ (\%) & & & $0.022^{*}$ \\
\hline$I \sim \|$ & $56(77.8)$ & $225(88.2)$ & \\
\hline III & $16(22.2)$ & $34(11.8)$ & \\
\hline Smoking history, n (\%) & $3 I(43 . I)$ & $98(33.9)$ & 0.147 \\
\hline Drinking history, n (\%) & $2 \mathrm{I}(29.2)$ & $74(25.6)$ & 0.539 \\
\hline Hypertension, n (\%) & $22(30.6)$ & $91(31.5)$ & 0.879 \\
\hline Diabetes, n (\%) & $9(12.5)$ & $35(12.1)$ & 0.928 \\
\hline BMI, $\mathrm{kg} / \mathrm{m}^{2}$ mean $(\mathrm{SD})$ & $23.03(3.33)$ & $24.43(3.54)$ & $0.003 *$ \\
\hline MMSE score, median (IQR) & $23(20,26)$ & $25(22,28)$ & $0.002^{*}$ \\
\hline $\mathrm{CCl}$ score, median (IQR) & $I(I, 2)$ & $I(0,2)$ & 0.106 \\
\hline PNI, mean (SD) & $45.5 I(6.74)$ & $49.91(5.76)$ & $<0.00 I^{*}$ \\
\hline Nutritional status (PNI), n (\%) & & & $<0.00 I^{*}$ \\
\hline $\mathrm{PNI}<40$ & $15(20.8)$ & $14(4.8)$ & \\
\hline PNI 40-45 & $20(27.8)$ & $35(12.1)$ & \\
\hline PNI $45-50$ & $17(23.6)$ & $91(31.5)$ & \\
\hline $\mathrm{PNI} \geq 50$ & $20(27.8)$ & $149(5 \mid .6)$ & \\
\hline \multicolumn{4}{|l|}{ Laboratory tests, mean (SD) } \\
\hline $\mathrm{Hb}$ & $116.78(22.10)$ & $130.39(18.05)$ & $<0.00 I^{*}$ \\
\hline Alb $(g / L)$ & $38.83(6.49)$ & $41.95(5.08)$ & $<0.00 I^{*}$ \\
\hline Lymphocyte count $\left(* 10^{9} / \mathrm{L}\right)$ & $I .4 I(0.60)$ & $1.63(0.59)$ & $0.006 *$ \\
\hline Potassium ions (mmol/L) & $3.97(0.35)$ & $4.03(0.46)$ & 0.338 \\
\hline Blood glucose (mmol/L) & $5.79(1.87)$ & $5.70(1.56)$ & 0.700 \\
\hline $\operatorname{ALT}(\mathrm{U} / \mathrm{L})$ & $23.58(34.90)$ & $24.68(29.01)$ & 0.748 \\
\hline $\mathrm{AST}(\mathrm{U} / \mathrm{L})$ & $25.85(26.76)$ & $25.5 \mathrm{I}(28.50)$ & 0.927 \\
\hline BUN (mmol/L) & $5.44(2.42)$ & $7.24(18.99)$ & 0.424 \\
\hline $\mathrm{Cr}(\mu \mathrm{mol} / \mathrm{L})$ & $64.69(16.88)$ & $63.42(15.56)$ & 0.540 \\
\hline Types of operation, $n$ (\%) & & & 0.357 \\
\hline Thoracic surgery & $16(22.2)$ & $89(30.8)$ & \\
\hline Abdominal surgery & $37(51.4)$ & $|3|(45.3)$ & \\
\hline Spinal, limb surgery & $19(26.4)$ & $69(23.9)$ & \\
\hline Blood transfusion, $\mathrm{n}(\%)$ & $7(9.7)$ & $15(5.2)$ & 0.150 \\
\hline \multicolumn{4}{|c|}{ Volume of blood and fluid transfusion, median (IQR) } \\
\hline Blood loss (mL) & $100(100,300)$ & $100(100,200)$ & 0.313 \\
\hline Urine $(\mathrm{mL})$ & $400(300,400)$ & $400(300,400)$ & 0.500 \\
\hline Crystalloid fluid $(\mathrm{mL})$ & $1225(1000,1500)$ & $1250(1000,1500)$ & 0.894 \\
\hline Hydroxyethyl starch (mL) & $500(50,500)$ & $500(350,500)$ & 0.444 \\
\hline Duration of surgery, mins median (IQR) & $153(100,239)$ & $160(110,210)$ & 0.640 \\
\hline Duration of anesthesia (min), median (IQR) & $188(\mid 25,270)$ & $188(125,270)$ & 0.646 \\
\hline
\end{tabular}

Notes: Data are expressed as the mean (standard deviation), median (interquartile range), or $n(\%)$; $* P<0.05$.

Abbreviations: POD, postoperative delirium; PNI, Prognostic Nutritional Index; CAM, Confusion Assessment Method; MMSE, Mini-Mental State Examination; CCl, Charlson Comorbidity Index; ASA, American Society of Anesthesiologists; BMI, body mass index; BUN, blood urea nitrogen; Cr, serum creatinine; ALT, alanine aminotransferase; AST, aspartate amino transferase; Hb, hemoglobin; Alb, albumin.

In addition, for the preoperative laboratory tests, the levels of blood lymphocyte count, hemoglobin and albumin were significantly lower in the POD group than the non-POD group ( $P<0.001)$. Furthermore, potassium ions, blood glucose, ALT, AST, BUN, Cr levels did not differ significantly between patients with and without $\operatorname{POD}(P>0.05)$. Compared 
Table 2 Risk Factors for POD by Univariable and Multivariable Logistic Regression Analyses

\begin{tabular}{|c|c|c|c|c|}
\hline & \multicolumn{2}{|c|}{ Univariable } & \multicolumn{2}{|c|}{ Multivariable } \\
\hline & $P$ & OR $(95 \% \mathrm{Cl})$ & $P$ & OR $(95 \% \mathrm{Cl})$ \\
\hline \multicolumn{5}{|l|}{ Model I } \\
\hline Age (I year increase) & $<0.001$ & 1.08 (1.04 to 1.13$)$ & 0.184 & $1.03(0.99$ to 1.08$)$ \\
\hline ASA (III vs IV) & 0.024 & 2.14 (1.10 to 4.15$)$ & 0.400 & $1.40(0.64$ to 3.09$)$ \\
\hline BMI $\left(1 \mathrm{~kg} / \mathrm{m}^{2}\right.$ increase $)$ & 0.003 & $0.89(0.82$ to 0.96$)$ & 0.064 & $0.92(0.85$ to 1.01$)$ \\
\hline MMSE (I point increase) & 0.004 & 0.90 (0.84 to 0.97$)$ & $0.034 *$ & $0.92(0.85$ to 0.99$)$ \\
\hline $\mathrm{Hb}$ (I g/L increase) & $<0.001$ & $0.97(0.95$ to 0.98$)$ & $0.029 *$ & $0.98(0.97$ to 0.99$)$ \\
\hline $\mathrm{CCl}$ (I point increase) & 0.089 & $1.23(0.97$ to 1.55$)$ & 0.160 & $1.20(0.93$ to 1.56$)$ \\
\hline PNI (I increase) & $<0.001$ & $0.89(0.85$ to 0.93$)$ & $0.008^{*}$ & $0.93(0.88$ to 0.98$)$ \\
\hline \multicolumn{5}{|l|}{ Model 2} \\
\hline Age (I year increase) & $<0.001$ & $1.08(1.04$ to 1.13$)$ & 0.098 & $1.04(0.99$ to 1.09$)$ \\
\hline ASA (III vs IV) & 0.024 & $2.14(1.10$ to 4.15$)$ & 0.474 & $1.34(0.60$ to 2.96$)$ \\
\hline BMI $\left(1 \mathrm{~kg} / \mathrm{m}^{2}\right.$ increase $)$ & 0.003 & $0.89(0.82$ to 0.96$)$ & 0.100 & $0.93(0.85$ to 1.01$)$ \\
\hline MMSE (I point increase) & 0.004 & $0.90(0.84$ to 0.97$)$ & $0.028 *$ & $0.92(0.85$ to 0.99$)$ \\
\hline $\mathrm{Hb}$ (I g/L increase) & $<0.001$ & $0.97(0.95$ to 0.98$)$ & $0.036 *$ & $0.98(0.97$ to 0.99$)$ \\
\hline $\mathrm{CCl}$ (I point increase) & 0.089 & $1.23(0.97$ to 1.55$)$ & 0.116 & $1.24(0.95$ to $\mathrm{I} .6 \mathrm{I})$ \\
\hline \multicolumn{5}{|l|}{ Nutritional status (PNI) } \\
\hline $\mathrm{PNI} \geq 50$ & & I (reference) & & I (reference) \\
\hline PNI 45-50 & 0.353 & $1.39(0.69$ to 2.79$)$ & 0.970 & $0.99(0.47$ to 2.09$)$ \\
\hline PNI 40-45 & $<0.001$ & $4.26(2.07$ to 8.75$)$ & $0.009 *$ & $2.92(1.31$ to 6.50$)$ \\
\hline $\mathrm{PNI}<40$ & $<0.001$ & $7.98(3.36$ to 18.96$)$ & $0.029 *$ & $3.15(1.12$ to 8.83$)$ \\
\hline
\end{tabular}

Notes: PNI was included in the univariable logistic regression model and the multivariable logistic regression model in two kinds of form (absolute value of PNI [Model I], classification of PNI [Model 2]) respectively; Variables with $P \leq 0.1$ in the univariable analysis were included in the multivariable regression model; $* P<0.05$.

Abbreviations: $\mathrm{Cl}$, confidence interval; OR, odds ratio.

with patients without POD, the PNI of patients with POD was lower; patients with moderate to severe malnutrition (PNI $40-44.9)$ and with serious malnutrition (PNI < 40) were more likely to develop POD (27.8\% versus $12.1 \%, 20.8 \%$ versus $4.8 \%$, respectively).

\section{Outcomes}

Univariable logistic regression analyses were performed to evaluate potential risk factors for POD. As shown in Table 2, 8 potential risk factors $(P<0.05$ in Table 1$)$ were included in the univariable logistic regression analysis. Collinearity diagnostics were evaluated for all variables; Alb and lymphocyte count were not incorporated in the model because of multicollinearity with PNI. Then, multivariable logistic regression was performed including 7 variables (age, ASA physical status, BMI, MMSE, PNI, CCI, Hb) with a $P$ value $\leq 0.1$ (2-sided) during the univariable analyses. All variance inflation factors were less than 10. PNI was included in the univariable logistic regression model and the multivariable logistic regression model in the form of absolute value of PNI and classification of PNI, respectively. In addition, Nagelkerke $\mathrm{R}^{2}$ of the multivariable logistic regression models were 0.213 and 0.226 (model1, model2) respectively. As a result, a statistical association was found between POD and PNI, MMSE and $\mathrm{Hb}$; PNI ( 1 point increase, $\mathrm{OR}=0.89,95 \% \mathrm{CI} 0.85-0.93, P=$ 0.008 ), MMSE (1 point increase, OR $=0.92,95 \%$ CI $0.85-$ $0.99, P=0.034)$ and $\mathrm{Hb}(1 \mathrm{~g} / \mathrm{L}$ increase, $\mathrm{OR}=0.98,95 \% \mathrm{CI}$ $0.97-0.99, P=0.029)$ were 3 independent risk factors for POD in elderly patients undergoing noncardiac surgery. Moderate to severe malnutrition $(\mathrm{OR}=2.92,95 \% \mathrm{CI} 1.31-6.50, P=0.009)$ and serious malnutrition (OR 3.15, 95\% CI 1.12-8.83, $P=$ 0.029 ) were associated with POD (Table 2).

\section{Cut-Off Values for PNI}

As shown in the ROC curve for the incidence of POD, the cut-off value for PNI according to the Youden index was 46.05. The area under the curve (AUC) was 0.692 (95\% CI $0.62-0.77)$. The sensitivity and specificity were 0.779 and 0.556 , respectively (Figure 2).

\section{Discussion}

This retrospective study was performed in a population of elderly patients undergoing elective noncardiac surgery in general anesthesia. We found that a preoperative lower value of PNI is significantly associated with the presence of POD. Furthermore, adjusted for age, ASA physical status, BMI, CCI, 


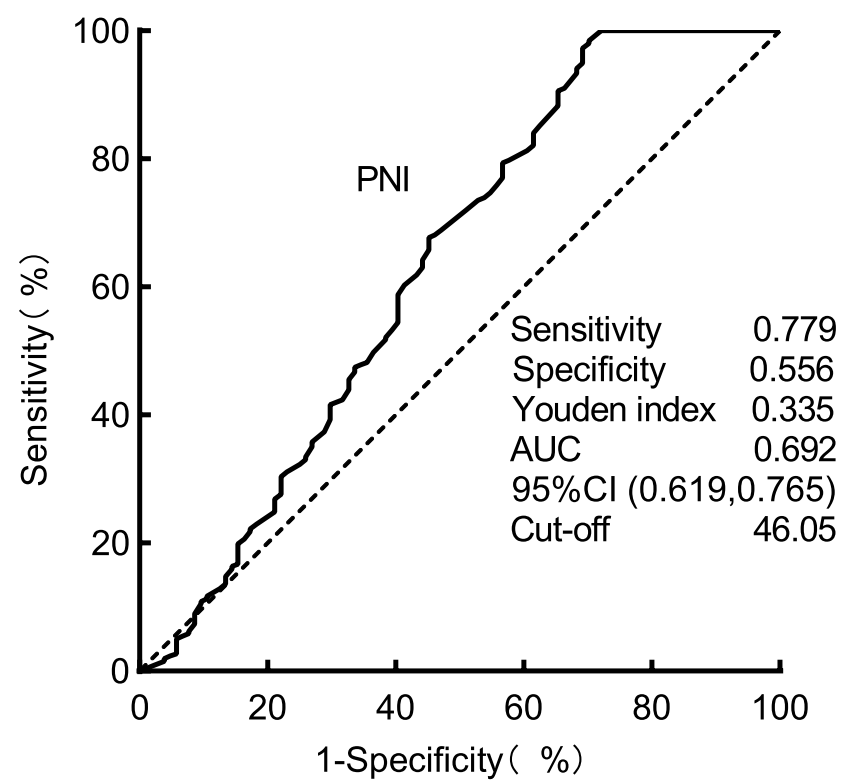

Figure 2 The ROC curve of PNI.

Abbreviations: PNI, Prognostic Nutritional Index; ROC, receiver operating characteristic curve; AUC, area under the curve.

hemoglobin and MMSE in a multivariable logistic regression analysis model, low value of PNI preoperatively was an independent risk factor for the development of POD. The risk of POD for individuals with moderate to severe malnutrition and serious malnutrition increased by 2.9 times and 3.1 times, respectively, compared with those with normal nutritional status.

Nutrition risk screening tools can be defined as subjective or objective assessment indicators. ${ }^{24}$ The subjective ones do not require special laboratory test and are easy to perform, but the accuracy is relatively low. Some subjective indicators, such as MNA-SF, are developed for white people and may be not suitable for other races. ${ }^{13}$ The objective indicators mainly consist of laboratory indicators, and thus are more accurate and expensive, such as PNI, CONUT and GNRI. A study compared the efficiency of GNRI, PNI and CONUT on the prediction of delirium in coronary ICU patients and found no significant difference between PNI and CONUT. ${ }^{25}$ However, PNI had the superiority in terms of convenience (CONUT is calculated by the serum albumin, serum total cholesterol and total lymphocyte counts).

Nowadays, preoperative malnutrition has been considered a predisposing factor for delirium. ${ }^{4,17,26}$ A study in patients undergoing spinal deformity surgery reported that PNI $<49.7$ predicted the incidence of POD. ${ }^{17}$ Another study of 163 elderly patients suggested that a low value of PNI is a predictor for POD in elderly patients after hip fracture surgery. ${ }^{16}$ However, for the present study, PNI was found to be a risk factor for POD after noncardiac surgery, and the PNI cut-off value for POD was 46.05 , which may enable medical caregivers to identify patients with malnutrition early. Our study is in line with these studies, though with the following differences. Firstly, the participants of the present study were noncardiac surgery patients. Secondly, previous studies focused on the relationship between the absolute value of PNI and POD, while this study assessed the nutrition status with the four-class stratification of the PNI tool and explored the correlation between different degrees of nutrition status and the risk of POD. Compared with patients with normal nutritional status, the risk of POD increased by 2.9 and 3.1 in patients with moderate to severe malnutrition and serious malnutrition, respectively. Thus, a more accurate and rapid assessment of the risk of POD could be done according to PNI values before surgery.

Malnutrition has been reported to be associated with several adverse effects, and numerous studies consistently showthat nutritional supplementation is helpful to reduce postoperative complications. $^{27,28}$ Improving the nutrition status during the perioperative period may be of great significance for the treatment and prevention of POD. The deficiency of nutrients is thought to contribute to the development of delirium and impair cognitive performance. ${ }^{29}$ A study in elderly hip-fracture patients demonstrated that metabolic abnormalities before surgery could possibly increase the vulnerability of the brain and result in POD, including lack of $\omega 3$ and $\omega 6$ fatty acids, dysfunction of energy metabolism and glutamate-glutamine cycle dysfunction. ${ }^{30}$ However another study in Irish older adults found that nutritional supplements of Omega-3 Polyunsaturated Fatty Acids and vitamin D could not improve overall cognitive function. ${ }^{31}$ In addition, a review reported that receiving high-protein nutritional supplements significantly improved the clinical outcomes of patients, reduced readmissions to hospital, and reduced surgical complications. ${ }^{28}$ Although there is no direct evidence demonstrating the benefits of nutritional supplements on preventing POD, it is hoped that this hypothesis can be verified.

Furthermore, the finding that lower preoperative hemoglobin level and lower MMSE scores are also risk predictors of POD is in line with the results of previous studies. ${ }^{32}$ The potential hypothesis is that the oxygenation function of blood decreases because of low levels of hemoglobin, and then the supply of energy and oxygen for brain tissue are limited, resulting in the disorder of cerebral metabolism and the onset of delirium symptoms such as disorientation and altered consciousness. The MMSE was used to screen for potential dementia before surgery. Similar to a previous study, ${ }^{33}$ the current study found that individuals with lower MMSE score 
before surgery had a greater risk of POD, and indicated that pre-existing cognitive impairment is associated with the development of POD.

The advantage of this study is that the sample size is relatively large and the study population contains most noncardiac major surgery, which strengthens the conclusion. In addition, seven protocols of postoperative followup eliminate a missed diagnosis of POD as far as possible.

However, this study has several possible limitations. Firstly, we only measured the incidence of POD on the first 3 days after surgery. Although POD regularly occurred during this period, it could occur at a later stage, so some patients with potential POD may be missed. Secondly, PNI may be affected by external factors and is not the most sensitive indicator for assessing the nutritional status of patients. This study only analyzed the relationship between preoperative PNI and POD, and failed to analyze the changes of postoperative PNI and its relationship with POD. Third, patients with dementia and pre-existing delirium were excluded, and there may be a decrease in the accuracy of PNI in predicting the occurrence of POD. In addition, the study was a single-center retrospective cohort study, the AUC of PNI (0.692) did not have enough accuracy, future prospective studies should be performed to validate these results, and the nomogram logistic analysis is helpful to quantify the contribution to the occurrence of POD.

\section{Conclusion}

In summary, for elderly patients undergoing noncardiac surgery, preoperative PNI value is associated with the development of POD. In addition, the risk of POD increases when the degree of nutritional inadequacy is more serious. Low hemoglobin and low MMSE scores are two other independent risk factors of POD.

\section{Abbreviations}

POD, postoperative delirium; PNI, Prognostic Nutritional Index; CAM, Confusion Assessment Method; MMSE, Mini-mental State Examination; CCI, Charlson Comorbidity Index; ASA, American Society of Anesthesiologists; BMI, body mass index; BUN, blood urea nitrogen; $\mathrm{Cr}$, serum creatinine; ALT, alanine aminotransferase; AST, aspartate amino transferase; Hb, hemoglobin; Alb, albumin.

\section{Data Sharing Statement}

The raw data set used/analyzed during the current study is available from Mingsheng Dai on reasonable request, and the data will be made available once the original research published.

\section{Author Contributions}

All authors made a significant contribution to the work reported, whether that is in the conception, study design, execution, acquisition of data, analysis and interpretation, or in all these areas; took part in drafting, revising or critically reviewing the article; gave final approval of the version to be published; have agreed on the journal to which the article has been submitted; and agree to be accountable for all aspects of the work.

\section{Funding}

This study was supported by the National Natural Science Foundation of China (81720108013, 31771161 to J.L. Cao; NSFC81300957 to H. Liu); Jiangsu Provincial Medical Youth Talent (QNRC2016795 to Y. Han), China Postdoctoral Science Foundation Funded Project (Project No. 2018T110556); the Natural Science Foundation of Jiangsu Province (BK20181145 to H. Liu), the Research Start-up Funding for Talent Introduction (2019203002 to H. Liu), and the Clinical Technical Research and Study Plan Project (2018211006 to H. Liu) .

\section{Disclosure}

The authors declare that they have no competing interests in this work.

\section{References}

1. Marcantonio ER. Delirium in hospitalized older adults. $N$ Engl J Med. 2017;377(15):1456-1466. doi:10.1056/NEJMcp1605501

2. van Eijk MMJ, Slooter AJC. Duration of ICU delirium, severity of the underlying disease, and mortality. Am J Respir Crit Care Med. 2010;181:419-420. doi:10.1164/ajrccm.181.4.419b

3. Bellelli G, Mazzola P, Morandi A, et al. Duration of postoperative delirium is an independent predictor of 6-month mortality in older adults after hip fracture. J Am Geriatr Soc. 2014;62(7):1335-1340. doi:10.1111/jgs. 12885

4. Aldecoa C, Bettelli G, Bilotta F, et al. European society of anaesthesiology evidence-based and consensus-based guideline on postoperative delirium. Eur J Anaesthesiol. 2017;34(4):192-214. doi:10.1097/ EJA.0000000000000594

5. Ali Abdelhamid Y, Chapman MJ, Deane AM. Peri-operative nutrition. Anaesthesia. 2016;71(Suppl 1):9-18. doi:10.1111/anae.13310

6. Cameron ID, Kurrle SE, Uy C, Lockwood KA, Au L, Schaafsma FG. Effectiveness of oral nutritional supplementation for older women after a fracture: rationale, design and study of the feasibility of a randomized controlled study. BMC Geriatr. 2011;11(1):32. doi:10.1186/1471-2318-1132

7. Olofsson B, Stenvall M, Lundström M, Svensson O, Gustafson Y. Malnutrition in hip fracture patients: an intervention study. $J$ Clin Nurs. 2007;16:2027-2038. doi:10.1111/j.1365-2702.2006.01864 
8. Tucker KL. Nutrient intake, nutritional status, and cognitive function with aging. Ann N Y Acad Sci. 2016;1367(1):38-49. doi:10.1111/ nyas. 13062

9. Leung JSL, Seto A, Li GKH. Association between preoperative nutritional status and postoperative outcome in head and neck cancer patients. Nutr Cancer. 2017;69(3):464-469. doi:10.1080/ 01635581.2017.1285406

10. Seretis C, Kaisari P, Wanigasooriya K, Shariff U, Youssef H. Malnutrition is associated with adverse postoperative outcome in patients undergoing elective colorectal cancer resections. $J$ BUON. 2018;23(1):36-41.

11. Malnutrition AG. A consistent and reliable tool for malnutrition screening. Nurs Times. 2003;99(46):26-27.

12. Ignacio De Ulíbarri J, González-Madroño A, de Villar NGP, et al. CONUT: a tool for controlling nutritional status. First validation in a hospital population. Nutr Hosp. 2005;20(1):38-45.

13. Rubenstein LZ, Harker JO, Salva A, Guigoz Y, Vellas B. Screening for undernutrition in geriatric practice: developing the short-form mini-nutritional assessment (MNA-SF). J Gerontol a Biol Sci Med Sci. 2001;56(6):M366-372. doi:10.1093/gerona/56.6.m366

14. Bouillanne O, Morineau G, Dupont C, et al. Geriatric nutritional risk index: a new index for evaluating at-risk elderly medical patients. $\mathrm{Am}$ J Clin Nutr. 2005;82(4):777-783. doi:10.1093/ajcn/82.4.777.

15. Onodera T, Goseki N, Kosaki G. Prognostic nutritional index in gastrointestinal surgery of malnourished cancer patients. Nihon Geka Gakkai Zasshi. 1984;85(9):1001-1005.

16. Xing H, Xiang D, Li Y, Ji X, Xie G. Preoperative prognostic nutritional index predicts postoperative delirium in elderly patients after hip fracture surgery. Psychogeriatrics. 2020;20(4):487-494. doi:10.1111/psyg.12511

17. Oe S, Togawa D, Yamato Y, et al. Preoperative age and prognostic nutritional index are useful factors for evaluating postoperative delirium among patients with adult spinal deformity. Spine. 2019;44 (7):472-478. doi:10.1097/Brs.0000000000002872

18. Wyrobek J, LaFlam A, Max L, et al. Association of intraoperative changes in brain-derived neurotrophic factor and postoperative delirium in older adults. Br J Anaesth. 2017;119(2):324-332. doi:10.1093/ bja/aex 103

19. Charlson M, Szatrowski TP, Peterson J, Gold J. Validation of a combined comorbidity index. J Clin Epidemiol. 1994;47 (11):1245-1251. doi:10.1016/0895-4356(94)90129-5

20. Kanda M, Fujii T, Kodera Y, Nagai S, Takeda S, Nakao A. Nutritional predictors of postoperative outcome in pancreatic cancer. Br J Surg. 2011;98(2):268-274. doi:10.1002/bjs.7305

21. Inouye SK, van Dyck CH, Alessi CA, Balkin S, Siegal AP, Horwitz RI. Clarifying confusion: the confusion assessment method. A new method for detection of delirium. Ann Intern Med. 1990;113 (12):941-948. doi:10.7326/0003-4819-113-12-941
22. Ely EW, Margolin R, Francis J, et al. Evaluation of delirium in critically ill patients: validation of the confusion assessment method for the intensive care unit (CAM-ICU). Crit Care Med. 2001;29 (7):1370-1379. doi:10.1097/00003246-200107000-00012

23. Avidan MS, Maybrier HR, Abdallah AB, et al. Intraoperative ketamine for prevention of postoperative delirium or pain after major surgery in older adults: an international, multicentre, double-blind, randomised clinical trial. Lancet. 2017;390(10091):267-275. doi:10.1016/S0140-6736(17)31467-8

24. van Bokhorst-de van der Schueren MAE, Guaitoli PR, Jansma EP, de Vet HCW. A systematic review of malnutrition screening tools for the nursing home setting. J Am Med Dir Assoc. 2014;15(3):171-184. doi:10.1016/j.jamda.2013.10.006

25. Sugita Y, Miyazaki T, Shimada K, et al. Correlation of nutritional indices on admission to the coronary intensive care unit with the development of delirium. Nutrients. 2018;10(11):1712. doi:10.3390/nu10111712

26. Mazzola P, Ward L, Zazzetta S, et al. Association between preoperative malnutrition and postoperative delirium after hip fracture surgery in older adults. J Am Geriatr Soc. 2017;65(6):1222-1228. doi:10.1111/jgs.14764

27. Schuetz P, Fehr R, Baechli V, et al. Individualised nutritional support in medical inpatients at nutritional risk: a randomised clinical trial. Lancet. 2019;393(10188):2312-2321. doi:10.1016/S0140-6736(18)32776-4

28. Cawood AL, Elia M, Stratton RJ. Systematic review and meta-analysis of the effects of high protein oral nutritional supplements. Ageing Res Rev. 2012;11(2):278-296. doi:10.1016/j. arr.2011.12.008

29. Sanford AM, Flaherty JH. Do nutrients play a role in delirium? Curr Opin Clin Nutr Metab Care. 2014;17(1):45-50. doi:10.1097/ MCO.0000000000000022

30. Guo Y, Zhang Y, Jia P, et al. Preoperative serum metabolites are associated with postoperative delirium in elderly hip-fracture patients. J Gerontol a Biol Sci Med Sci. 2017;72(12):1689-1696. doi:10.1093/gerona/glx001

31. Moran C, Scotto Di Palumbo A, Bramham J, et al. Effects of a six-month multi-ingredient nutrition supplement intervention of omega-3 polyunsaturated fatty acids, vitamin $\mathrm{D}$, resveratrol, and whey protein on cognitive function in older adults: a randomised, double-blind, controlled trial. J Prev Alzheimers Dis. 2018;5 (3):175-183. doi:10.14283/jpad.2018.11

32. Chen YL, Lin HC, Lin KH, et al. Low hemoglobin level is associated with the development of delirium after hepatectomy for hepatocellular carcinoma patients. PLoS One. 2015;10(3):e0119199. doi:10.1371/journal.pone.0119199

33. Price CC, Garvan C, Hizel LP, Lopez MG, Billings F. Delayed recall and working memory MMSE domains predict delirium following cardiac surgery. $J$ Alzheimers Dis. 2017;59(3):1027-1035. doi:10.3233/JAD-170380
Risk Management and Healthcare Policy

\section{Publish your work in this journal}

Risk Management and Healthcare Policy is an international, peerreviewed, open access journal focusing on all aspects of public health, policy, and preventative measures to promote good health and improve morbidity and mortality in the population. The journal welcomes submitted papers covering original research, basic science, clinical \& epidemiological studies, reviews and evaluations, guidelines, expert opinion and commentary, case reports and extended reports. The manuscript management system is completely online and includes a very quick and fair peer-review system, which is all easy to use. Visit http://www.dovepress.com/testimonials.php to read real quotes from published authors. 